Michel Eyquem de Montaigne (1533-1592)

François de La Rochefoucauld (1613-1680) 
Bereitgestellt von | De Gruyter / TCS

Angemeldet

Heruntergeladen am | 30.01.20 13:14 


\section{Montaigne und La Rochefoucauld: Emotionen in der Moralistik}

\section{Markus Wild}

\section{Wer und was sind die Moralisten?}

Als „Moralisten“ werden eine Reihe französischer Schriftsteller und Philosophen des 16. bis 18. Jahrhunderts bezeichnet. ${ }^{1} \mathrm{Zu}$ den klassischen Autoren der französischen Moralistik gehören Michel de Montaigne (15331592), François de La Rochefoucauld (1613-1680), Blaise Pascal (16231662) und Jean de La Bruyère (1645-1696). ${ }^{2}$ Die Moralisten gelten als Autoren je eines Buchs, das zugleich eine je neue literarische Form erschafft: Montaigne ist Autor und Erfinder der Essais (1580), La Rochefoucauld der Maximes (1665), Pascal der Pensées (posthum 1670) und La Bruyère der Charactères (1688). Äußerlich lassen sich die Moralisten an diesen literarischen Formen erkennen. Bei ihren Werken handelt es sich um Sammlun-

1 Die Moralistik ist jedoch ein über den französischen Kontext hinausweisendes Phänomen, denn sie knüpft sowohl bei Denkern der Antike an, insbesondere bei den Stoikern, als auch bei Autoren der italienischen Renaissance wie Machiavelli oder Castiglione. Moralistische Autoren finden sich in Spanien - etwa in der Gestalt von Baltasar Gracián - oder in England, vertreten durch Francis Bacon; vgl. Balmer 1981, 69-85; Zimmer 1999, 24-35, 47-69. In Deutschland wurde diese moralistische Tradition durch Lichtenberg, Schopenhauer, Nietzsche sowie Canetti aufgenommen und weitergeführt; vgl. Kruse 2003, 246-262; Balmer 1981, 127-180; Zimmer 1999, 117-155. Die Tradition der Moralistik wurde in der französischen Aufklärung fortgesetzt, die literarischen Formen erweitert. Exemplarisch: Nicolas Chamforts Maximes et pensées, charactères et anecdotes (franz. zuerst 1796).

2 Die Bezeichnung geht auf die 1820 erschienene Collection de Moralistes francais von Amaury Duval zurück. Eine neue Textsammlung ist Lafond 1992. Gesamtdarstellungen zur Moralistik bieten Balmer 1981, Stackelberg 1982, Zimmer 1999, Parmentier 2000. In French Moralists. The Theory of the Passions 1585-1649 (Levi 1964) behandelt Levi weitere und anders orientierte Autoren, als die klassischen Moralisten (etwa Franz von Sales, Jean-Pierre Camus, Nicolas Coëffeteau oder Marin Cureau de la Chambre), sodass seine Thesen die Moralistik im engeren Sinne aus den Augen verlieren. 
gen vorwiegend kurzer Textformen, die auf den ersten Blick unsystematisch und wie zufällig angeordnet wirken. ${ }^{3}$ Margot Kruse bringt wesentliche Züge der Moralistik auf den Punkt:

Die französischen Moralisten sind nicht Moralphilosophen oder Schriftsteller, die die Moral ihrer Zeit in normativer Absicht kritisieren, sondern Autoren, die die Sitten der Menschen beobachten, ihr eigenes Verhalten und das ihrer Umwelt analysieren. Über das Wesen des Menschen und die Motive seines Handelns nachdenken und ihre Reflexionen in unsystematischer, dem Gegenstand der Beobachtung angemessener Form zur Darstellung bringen. ${ }^{4}$

Descartes zog es vor, eher im großen Buch der Welt als in Büchern zu lesen, Moralisten ziehen dem Bücher- das Menschenstudium vor. ${ }^{5}$ Im Fokus steht der Mensch in seinen Selbst- und Fremdverhältnissen. ${ }^{6}$ Moralisten hegen Zweifel am Bild des Menschen von sich selbst. Ohne große Abstriche kann auf sie jene Formel angewendet werden, die Paul Ricoeur auf Marx, Nietzsche und Freud angewendet hat: „Meister des Verdachts“.7 Das Bewusstsein der Menschen (von sich und anderen) wird als falsches Bewusstsein verdächtigt. Die Verdachtsmeister führen gleichsam den cartesischen Zweifel weiter. Am Anfang der Meditationen zweifelt Descartes an den Objekten der Außenwelt: Es könnte sein, dass die gesamte Außenwelt eine große Täuschung ist. Und es ist mit einiger Sicherheit so, dass die Objekte ganz anders beschaffen sind, als sie unseren Sinnen erscheinen. Die Moralisten nun wenden diesen Zweifel auf das Bild des Menschen von sich selbst an: Es könnte sein, dass dieses Bild Selbsttäuschung ist. Und es ist mit einiger Sicherheit so, dass wir uns ganz anders erscheinen, als wir tatsächlich sind. So sind Moralisten grundsätzlich skeptisch. ${ }^{8}$ Eine Tendenz zu demaskieren, zu decouvrieren und zu desillusionieren steht im Vordergrund. ${ }^{9}$ La Rochefoucauld etwa führt Charaktereigenschaften, Tugenden oder Leidenschaften auf das basale Faktum der Eigenliebe (amour-propre) zurück. Bei Montaigne erscheint der Mensch als

3 Zu den Textformen vgl. Parmentier 2000, 199-260.

4 Kruse 2003, 1.

5 Vgl. Descartes' Einleitung zum Discours de la méthode. La Rochefoucauld schreibt: „Il est plus nécessaire d'étudier les hommes que les livres.“/„Es ist nötiger die Menschen zu studieren als die Bücher.“ (M 550)

6 Vgl. Stackelberg 1982, 7; Balmer 1981, $11 \mathrm{f}$.

7 Ricoeur 1993 (franz. zuerst 1965), 45ff.; vgl. Campion 1998.

8 Stackelberg 1982, 8.

9 Das Motto von La Rochefoucaulds Maximes lautet: „Nos vertus ne sont le plus souvent que des vices déguisés.“/„Unsere Tugenden sind häufig nichts als verkleidete Laster." Vgl. Balmer 1981, 89ff.; Clark 1994; Campion 1998. 
schwaches, eitles, unstetes Wesen, das seine Schwächen kompensieren muss und diese Kompensationen irrigerweise für Stärken ansieht. In beiden Fällen verhüllt der Mensch seine tatsächlichen Antriebe (Eigenliebe und Schwäche) vor sich und seinesgleichen. Das dabei entstehende Bild ist häufig wenig schmeichelhaft, erhebt aber den Anspruch eines augenöffnenden und von Selbsttäuschungen befreienden Realismus hinsichtlich der menschlichen Verhaltensweisen. Anders als Kruse meint, verfolgen die Moralisten also durchaus kritische Absichten.

Im Zentrum der moralistischen Beobachtung steht der Mensch als ein Wesen, das sich selbst unter seinesgleichen beobachtet. Darin bilden die „passions“"10 einen wichtigen Fokus, wobei im Vordergrund die Frage der Rolle von Emotionen in Selbst- und Fremdverhältnissen steht. ${ }^{11}$ Dabei treten genetische, systematische oder klassifikatorische Gesichtspunkte zurück hinter die Wandelbarkeit von Emotionen und deren Autonomie gegenüber kognitiven Zuständen. Das methodische Vorgehen der Moralisten besteht darin, dass sie von der Analyse komplexer Einzelfälle ausgehen und daran anschließend implizite Emotionstheorien entwickeln. Die Moralisten stehen im Gegensatz zu der in vielen gegenwärtigen Emotionstheorien vorherrschenden Tendenz zu einer „Überintellektualisierung“"12 von Emotionen, der zufolge Emotionen als „kognitive Zustände“ aufgefasst, auf „Überzeugungen“ zurückgeführt und mit „Rationalität“ geadelt werden. Doch erstens können Emotionen mehr sein als rational oder irrational, sind sie doch (un-)angemessen, (un-)verhältnismäßig oder auch (un-)verständlich. Zweitens besteht das Charakteristische an Emotionen primär darin, dass sie sich auf eine bestimmte Weise anfïblen; sie haben einen phänomenalen Charakter. ${ }^{13}$ So suchen oder meiden wir bestimmte Emotionen, weil sie sich auf bestimmte Weise anfühlen. Dies bedeutet nicht, dass Emotionen keine kognitiven Aspekte hätten, sondern, dass der phänomenale Charakter für sie wesentlich ist. ${ }^{14}$ Moralisten wie Montaigne

10 Ich übersetze passion mit „Emotion“. „Leidenschaft“ wäre als Übersetzung angemessen, bezeichnet im heutigen Sprachgebrauch jedoch entweder ein heftiges Gefühl oder ein intensives Hobby.

11 Vgl. Levi 1964, 4.

12 Der Ausdruck stammt von Goldie 2000, 3. Zur Kritik kognitiver Ansätze vgl. Griffiths 1997, 21-43.

13 Vgl. Goldie 2000, 50-83.

14 So auch David Hume, der Emotionen zuerst hinweisend über deren phänomenalen Charakter definiert, um dann die kausalen und kognitiven Elemente des Stolzes herauszuarbeiten; vgl. den Beitrag zu Hume in diesem Band. 
oder La Rochefoucauld heben den phänomenalen Charakter der Emotionen und deren Autonomie hervor.

Im Folgenden kann es nicht darum gehen, die Emotionstheorien der vier klassischen Moralisten vorzustellen, deshalb werde ich mich auf zwei Exponenten beschränken. Montaigne gilt als Gründerfigur der Moralistik. In Abschnitt 2 wird exemplarisch seine Behandlung des Zorns vorgestellt. Abschnitt 3 widmet sich der „,reinste[n] Verkörperung der Moralistik“"15, nämlich La Rochefoucaulds Behandlung der Emotionen und ihrer Beziehungen zur Eigenliebe. Abschließend soll auf die Relevanz der Moralisten in der Geschichte der Emotionstheorien verwiesen und ein ihrer Betrachtungsweise der Emotionen impliziter theoretischer Vorschlag offengelegt werden, dem zufolge Emotionen so etwas wie Handlungen sind.

\section{Montaigne: Der Zorn als exemplarische Emotion}

Montaigne wird mit Recht als Skeptiker betrachtet, ${ }^{16}$ denn in seinen Augen ist der Mensch ein ,wahnhafter, widersprüchlicher und hin und her schwankender" (E I, 1, 9/10) Gegenstand, über den sich keine festen Urteile fällen lassen. Dies drückt sich auch in der unsystematischen Form der Essais aus. Dieses Werk besteht aus 107 Einzelessays, deren Themen und Umfang beträchtlich variieren. ${ }^{17}$ Was hält dieses Buch zusammen? Montaigne erklärt: „Ich selbst, Leser, bin also der Inhalt meines Buchs“ (E 3/5). Die Essais sind der Versuch einer Selbstdarstellung. Noch weniger als dem wechselhaften Gegenstand ,Mensch“ kann eine systematische Darstellungsform einem Einzelnen angemessen sein. Aus diesen Gründen liefert Montaigne auch keine Emotionstheorie. Dennoch können die wichtigen Punkte seiner Behandlung der Emotionen exemplarisch an einer Emotion aufgezeigt werden, der ein eigener Essay gewidmet ist: „Über den Zorn“ (E II, 31). ${ }^{18}$

15 Stackelberg 1982, 4.

16 Wild 2006, 44-66.

17 Die ersten beiden Bücher erscheinen 1580. In einer erweiterten Auflage von 1588 fügt Montaigne ein drittes Buch und Zusätze zu den bereits vorhandenen Texten hinzu. Bis zu seinem Tod ergänzt und verändert er seinen Text in seinem Handexemplar, dem sog. „Exemplaire de Bordeaux“.

18 Weitere Essays handeln von der Trauer (E I, 2), von der Macht der Emotionen (E I, 3), von der Suche der Emotionen nach neuen Objekten (E I, 3), von der Angst (E I, 18), vom Hochmut (E II, 17) und von der Reue (E III, 2). 
Um Montaignes Behandlung des Zorns zu verstehen, ist es jedoch erforderlich, Eckpunkte seiner moralphilosophischen Konzeption darzustellen. Worin besteht der philosophische Nutzen einer Selbstdarstellung? An einer berühmten Stelle schreibt Montaigne:

Die anderen bilden den Menschen, ich bilde ihn ab [...]. Ich führe ein Leben ohne Glanz und Gloria vor Augen [Je propose une vie basse et sans lustre] - warum auch nicht? Man kann alle Moralphilosophie ebensogut auf ein niedriges und namenloses wie auf ein reicher ausgestattetes Leben gründen: Jeder Mensch trägt die ganze Gestalt des Menschen in sich [chaque homme porte la forme entiere de l'bumaine condition]. (E III, 2, 804f./398f.)

Der Grundgedanke lautet, dass sich die ganze Form der menschlichen Lebensbedingungen in einem gewöhnlichen Individuum finden lasse, das gerade in seiner Gewöhnlichkeit exemplarisch sei. Wie ist das zu verstehen? Montaigne richtet sich gegen die perfektionistische Moral des Weisen, der Asketen, Märtyrer, Krieger und seiner adeligen Standesgenossen. Diese folgen einer „Ethik der inneren Regierung“, die gegenüber äußeren Umständen und inneren Anfechtungen die Kontrolle bewahrt. Selbstbeherrschung ist das Charakteristikum dieser Ethik. ${ }^{19}$ In dem wichtigen Essay „Über die Grausamkeit“ (E II, 11) findet eine Verschiebung des moralischen Fokus von der Selbstbeherrschung zu den Handlungsfolgen statt. ${ }^{20}$ Der zentrale Satz lautet: „Von Natur wie auch aus Vernunftgründen habe ich einen grausamen Hass auf die Grausamkeit, denn sie ist für mich das äußerste aller Laster.“ (E II, 11, 429/168) Dieser Essay beginnt mit der Betrachtung einer Ausprägung der Ethik innerer Regierung, nämlich des tugendhaften Lebens. Tugenden werden als Fähigkeiten zur Beherrschung von Emotionen oder Wünschen betrachtet. Sokrates und Cato d. J. sind Musterbilder tugendhaften Lebens. Ihnen unterstellt Montaigne Grausamkeit gegen sich selbst. Ist aber Grausamkeit das Schlimmste, sollte uns die gegen sich selbst grausame Selbstbeherrschung nicht als Vorbild dienen. Höchste Selbstbeherrschung ist einem „Leben ohne Glanz und Gloria“ nicht nur unangemessen, sondern sie ist moralisch verwerflich, eben weil sie Ausdruck von Grausamkeit gegenüber sich selbst ist. Mon-

19 Wie viele seiner Zeitgenossen denkt Montaigne hierbei an den durch Seneca vermittelten Stoizismus. Der einflussreiche Neostoiker Justus Lipsius (15471606) fasst die Ethik der inneren Regierung als constantia und besimmt diese als „rechtmesige unnd unbewegliche stercke des gemüts/ die von keinem eusserlichen oder zufelligen dinge erhebt oder untergedrueckt wird." (De constantia I, 4, Lipsius 1965 (lat. zuerst 1583/84), 10)

20 Vgl. Hallie 1977, von dem der Ausdruck „Ethik der inneren Regierung“ (Inward Government Ethics) stammt. 
taigne verschiebt den Fokus von der inneren Regierung auf die Folgen für empfindungsfähige Lebewesen. Das Mitgefühl tritt an die Stelle der Selbstbeherrschung. Montaigne schreibt im Zeitalter der Religionskriege, inmitten exzessiver Gewalt und Grausamkeit. In diesem Kontext ist sein Plädoyer für die Einübung in das Mitgefühl zu sehen. ${ }^{21}$ Damit schreibt er auch unter den Bedingungen des Verlusts einer verbindlichen moralischen Grundlage des öffentlichen Lebens. Lebensformen und deren moralische Leitvorstellungen erweisen sich als Ergebnisse lokaler Gewohnheiten und Praktiken (vgl. E II, 23). Demzufolge sind moralische Leitvorstellungen keine göttlichen oder natürlichen Satzungen. Sie entstammen der Praxis und müssen sich an der Praxis bewähren. Die Regel lautet: Frage dich stets, ob sich einer moralischen Leitvorstellung gemäß überhaupt leben lasse und ob sie mit deinen gewöhnlichen Emotionen, Bedürfnissen und Überzeugungen vereinbar sei. ${ }^{22}$ Mit moralischen Leitvorstellungen werden Versuche angestellt. Diese dürfen subjektiv sein und sich auf die Selbstdarstellung konzentrieren, denn „chaque homme porte la forme entiere de l'humaine condition" (E III, 2, 805/399). Damit ist nicht gemeint, dass jeder Mensch die Essenz des Menschen in sich trage, sondern dass jeder vergleichbaren Bedingungen ausgesetzt ist (der bumaine condition). Die Lebensform, die sich bei jemandem ausprägt, ist subjektiv, aber deshalb nicht subjektivistisch, denn sie kann sich von den Bedingungen, in denen sich gewöhnliche Menschen finden, nicht allzu weit entfernen. Natürlich spielen nun Emotionen in diesem Bild eine wichtige Rolle.

Schaut doch einmal, was die gewöhnliche Erfahrung uns zeigt: Da ist keiner, der, falls er sich ausforscht, nicht in sich eine ihm eigne Form entdeckte, eine Grundform [une forme sienne, une forme maistresse], die sich gegen die Erziehung und gegen den Ansturm all der Emotionen zu behaupten sucht, die ihr feind sind. (E III, 2, $811 / 402)$

Im Rückgriff auf das Gesagte kann diese Rolle unter vier Aspekte gebracht werden: (i) In einer Ethik der inneren Regierung zielen moralische Leitvorstellung auf Emotionskontrolle. (ii) Emotionen sind ein wichtiger Bestandteil der gewöhnlichen condition bumaine. (iii) Sie sind in die Struktur von Einzelleben eingewoben. (iv) Sie können gravierende Folgen für andere und für uns selbst haben. Insbesondere der letzte Punkt, die Folgen der Emotionen für den Selbstbezug, treiben Montaig-

21 Vgl. Quint 1998, 134: „Montaigne’s preference for clemency over justice [...] is a dominant ethical strain in his book."

Vgl. Schneewind 1998, 44ff. 
nes Auseinandersetzung mit dem Zorn um. Von welchen Voraussetzungen geht Montaigne aus?

Zorn ist seit Senecas De ira das Paradigma einer zu kontrollierenden Emotion. Im Anschluss an Aristoteles betrachtet Seneca den Zorn als Wunsch, erlittene Kränkungen zu rächen. ${ }^{23}$ Aristoteles bemerkt präziser, dass es sich um eine zu Unrecht erlittene Kränkung an einem selbst oder nahe stehenden Personen handelt, die den Wunsch nach Vergeltung weckt. $^{24}$ Zorn ist eine komplexe kognitive und soziale Emotion, die Urteile und ein soziales Gefüge involviert. Zugleich bedroht der Zorn unser Urteilsvermögen und das soziale Gefüge und bedarf deshalb der Kontrolle. Zorn, so Seneca, darf keinesfalls Bestandteil einer philosophischen Lebensführung sein, er sollte ganz aus dem Leben der Menschen ausgemerzt werden. Anders als Seneca hält Aristoteles den Zorn nicht für ganz und gar schädlich, befördert er doch die Tapferkeit.

Montaigne weist diese klassische Definition nicht ausdrücklich zurück, noch bestreitet er die Destruktivität des Zorns. Anders als die beiden antiken Autoren betont er aber die Autonomie der Emotion gegenüber ihren kognitiven und sozialen Aspekten. Betrachten wir Montaignes erstes Beispiel für den Zorn:

Wenn ich zum Beispiel durch unsre Straßen ging und beobachtete, wie kleine Jungen von einem wutschnaubenden Vater, einer irrsinnig tobenden Mutter grün und blau geschlagen und zu Boden geprügelt wurden - wie oft packte mich da die Lust, mit bühnenreifem Donnerwetter dreinzufahren und den Ärmsten zu rächen [de dresser une farce, pour venger des garconnet:]! Man sieht solche Eltern blitzenden Auges, ja geradezu feuerspeiend [sortir le feu et la rage des yeux] über ihre Sprößlinge herfallen $[\ldots]$ und mit schneidender, weithin gellender Stimme auf ein Kind einbrüllen [à tout une voix tranchante et esclatante], das häufig gerade erst der Ammenbrust entwöhnt ist. (E II, 31, 714/353)

Das Beispiel könnte durchaus innerhalb der klassischen Definition des Zorns untergebracht werden: Der Beobachter der Misshandlung gerät in Rage, weil er glaubt, dem Kind werde unangemessen und unangebracht Übel mitgespielt. Es gibt jedoch Unterschiede, und gerade in diesen Unterschieden liegt, was für Montaignes Auffassung der Emotionen kennzeichnend ist. Ein erster Unterschied besteht darin, dass ein Kleinkind kein direktes Objekt der Vergeltung sein kann. Schwer vorzustellen, womit das Kind seine Eltern ungerechtfertigt gekränkt haben könnte, worauf deren Rachewunsch beruht. Der ungehemmte elterliche Zorn scheint un-

23 Seneca, De ira 1, 3, 3.

24 Aristoteles, Rhetorik II.2, 1378a, 31-33. 
angemessen und unverhältnismäßig, die Handlungsfolgen - die Kinder sind „körperlich und geistig zu Krüppeln geschlagen“ (ebd.) - gravierend. Sich selber setzt Montaigne als jemanden ins Bild, der über die Eltern in Zorn gerät und den Misshandelten rächen möchte. Doch der Junge ist ihm fremd, er steht ihm nicht nah. Über den elterlichen Zorn soll auch der Leser in Zorn geraten. Dies ist ein zweiter wichtiger Unterschied: der Zorn der Zeugen über den elterlichen Zorn entspringt keiner besonderen sozialen Beziehung zum Kleinkind, sondern einem generalisierten Mitgefühl.

Ein dritter Aspekt, den Montaigne betont, liegt in dem Umstand, dass Zorn ansteckt. Dies wurde freilich bereits von Seneca betont, der darin eine der Hauptgefahren dieser Emotion erkannte. Montaigne fasst diesen Umstand jedoch stärker deskriptiv, nämlich als Bestandteil von Emotionen überhaupt. Die Ansteckung erfolgt nur teilweise über die Beurteilung der Situation, hier werde jemand unangemessen und unangebracht gemaßregelt. Wichtiger ist, was man „mimetische Übertragung“ nennen könnte. Sie erfolgt durch expressive, körperliche Manifestationen einer Emotion („Wutschnauben“, „das blitzende Auge“, die „schneidende, gellende Stimme“ usw. (E II, 31, 714/353)). Menschen neigen dazu, diese äußerlichen Manifestationen einer Emotion spontan zu imitieren, durch die körperliche Imitation wird die Emotion dann übertragen (oder eine Gegenemotion hervorgerufen). Nicht nur traurige, ekelerregende oder schockierende Dinge erwecken in uns Trauer, Ekel oder Schrecken, sondern auch ein trauriger, angeekelter oder schockierter Ausdruck vermag diese Emotionen hervorzurufen. Es scheint sogar so, als würden Emotionen in der Individualentwicklung auf diesem Weg übernommen und erlernt, bevor wir über traurige, eklige oder schockierende Dinge überhaupt Bescheid wissen.

Auch in der Innenperspektive des Subjekts finden sich körperliche Manifestationen, jedoch qualitative (Hitze im Gesicht, Enge in der Brust, rasender Puls, kurz: innere Bewegung). Sowohl im sozialen Raum als auch im Subjekt wird eine Emotion durch ihre je spezifischen Manifestationen (die expressiven und qualitativen körperlichen Aktualisierungen) übertragen. Durch Manifestationen werden Emotionen wie der Zorn mimetisch übertragen. ${ }^{25}$ Dies führt uns zu einem begrifflichen Aspekt. Montaigne schreibt, Zorn sei

25 Montaigne beschreibt die Verbreitung und Übertragung von Angst in E I, 47, $285 / 144$. 
[...] eine Leidenschaft, die an sich selbst Gefallen findet und sich immer weiter anspornt [qui se plaist en soy et qui se flatte]. Wie oft richten wir, wenn wir uns aus falschem Grund ereifert haben und man uns zum Beweis eine triftige Entschuldigung und Rechtfertigung vorbringt, unseren ganzen Ärger nun sogar gegen die Wahrheit und die Unschuld! (E II, 31, 717/354)

Anders als in der klassischen Definition kann der Zorn nicht auf Urteile (über soziale Kränkungen) und Wünsche (nach Vergeltung) zurückgeführt werden. Zorn ist durch expressive und qualitative körperliche Manifestationen bestimmt, er erhält sich in diesen Manifestationen über die Revision von Urteilen hinweg. Er hat ihnen gegenüber Autonomie. Dies bedeutet nicht, dass Zorn allein durch seine Manifestationen bestimmt wäre. Wie zu anderen Emotionen gehört zum Zorn ein (intentionales) Objekt. Ist er aber erst einmal entstanden, wird ihm das Objekt sozusagen gleichgültig. Die durch Manifestationen bestimmte Emotion sucht sich ein Objekt, auf das sie sich richten kann. Emotionen weiten den Objektbereich aus, sie schränken ihn nicht ein. ${ }^{26}$ Emotionen finden ein beliebiges Objekt, auf das sie sich richten können, wenn sie erst einmal entstanden sind. Dem Ängstlichen erscheint in der dunklen, abgelegenen, unbekannten Straße alles gefährlich, dem Übellaunigen in seinem Zimmer alles unnütz und widerspenstig. Im sozialen Raum erhalten sich Emotionen durch die mimetische Übertragung expressiver, im Subjekt durch die innere Übertragung phänomenaler Manifestationen und die Projektion auf äußere Gegenstände.

Dennoch spricht sich Montaigne für Nachlässigkeit gegenüber zornigen Regungen aus. Eine Konsequenz seiner Zurückweisung der Ethik der inneren Regierung besteht nämlich darin, auch innere Konflikte zurückzuweisen. ${ }^{27}$ Es gibt keinen Konflikt zwischen Emotionen und Selbstbeherrschung, wenn die emotionalen Manifestationen gezielt zugelassen werden. Wie ist das $\mathrm{zu}$ verstehen? Montaigne verweist auf den ,zornigsten Menschen Frankreichs" (vermutlich Henry IV.), der sich aber stets beherrsche, und bemerkt:

Ich für mein Teil wüsste keine Leidenschaft, die zu zügeln und im Zaum zu halten ich eine derartige Anstrengung unternehmen könnte [pour laquelle couvrir et soustenir je peusse faire un tel effort - so teuer möchte ich mir die Weisheit nicht erkaufen! Ich sehe bei diesem Mann weniger darauf, was er tut, als darauf, wieviel es ihn kostet, nichts Schlimmeres zu tun. (E II, 31, 718f./355)

26 Anders als die in der gegenwärtigen Diskussion beliebte „Suchhypothese“, der zufolge Emotionen die Funktion zukommt, den Bereich möglicher Handlungsoptionen einzuschränken und die einzelnen Optionen zu gewichten; vgl. Evans 2002.

27 Vgl. Über die Reue (E III, 2). 
Es sei nicht empfehlenswert, sich innerlich zernagen zu lassen (se ronger interieurement), nur um den Anschein der Ruhe zu bewahren, man verleibe sich den Zorn sonst ein (on incorpore la cholere en la cachant). Besser sei es, seine Spitze nach außen zu richten, als in uns einzufalten (il vaut mieux que leur poincte agisse au dehors que de la plier contre nous) (ebd.). Montaigne zieht mithilfe von Metaphern wie „nagen“, „einverleiben“, „einfalten“ eine Konsequenz aus den Thesen der mimetischen Übertragung und der körperlichen Manifestationsweisen. Da die Emotion sich im Subjekt erhält, überträgt und nach neuen Objekten sucht, wird sie für das Subjekt schädlich. Wer den Zorn unterdrückt, zeitigt schädliche Folgen für sich und ist gegenüber sich selbst grausam.

Wie gesagt, richtet Montaigne die moralische Aufmerksamkeit von der inneren Regierung auf die Handlungsfolgen und auf das Mitgefühl. Als keine durchwegs natürliche Einstellung, muss Mitgefühl eingeübt werden. Eine Einübung sieht Montaigne in der Zulassung von Emotionen, die uns überkommen und die deshalb als nicht zu uns gehörig erscheinen. Dennoch mag auf den ersten Blick Montaignes Plädoyer für eine gezielte ÄuBerung zorniger Regungen überraschen:

Ich würde raten, lieber seinem Diener etwas zur Unzeit eine Backpfeiffe zu geben, als sich innerlich Zwang anzutun, nur damit man solch weises Gehabe [cette sage contenance] an den Tag legen kann. Ich zöge es jedenfalls vor, meine Leidenschaften offen hervorbrechen als zu meinem Schaden in mir schwelen zu lassen. Sobald sie sich Luft verschaffen und entladen, sinken sie kraftlos zusammen [elles s'alanguissent en s'esvantant et en s'exprimant. (E II, 31, 719/355)

Sind hier nicht sowohl die Erhaltung der Emotion als auch die Handlungsfolgen und das Mitleid vergessen? Nein. Montaigne gibt den Folgen für das Subjekt des Zorns den Vorrang vor dem Opfer. Dass sich Emotionen in ihren Manifestationen erhalten, bedeutet nicht, dass sie nicht abklingen können, die gerielte Entladung unterbricht die Erhaltung und die unkontrollierte Übertragung. Im Anschluss an die zitierte Stelle erteilt Montaigne drei Ratschläge: Man solle mit Zornausbrüchen sparsam umgehen, sich direkt auf das Objekt des Zorns beziehen, sich bei triftigen Anlässen in Zurückhaltung üben. Schließlich weist er darauf hin, dass er um der Ordnung seines Hauswesens willen hin und wieder den Wutentbrannten spielt (E II, 31, 719f./355f.). Er plädiert für eine gezielte Zulassung von Emotionen (vgl. E III, 13). Die gezielte Zulassung der Emotionen ist mehr als ein bloßes Abreagieren, sondern eine geradezu instrumentelle Verwendung von emotionalen Zuständen. An dieser Stelle zeichnet sich ein Gedanke ab, den La Rochefoucauld aufnimmt, und den wir im 
vierten Teil dieses Essays explizieren werden: Emotionen weisen Parallelen mit Handlungen auf.

In diesem gezielten und geradezu dramaturgischen Umgang mit Emotionen finden sich Reste der Ethik der inneren Regierung. Am Schluss seines Essays kritisiert Montaigne die aristotelische These, der Zorn befördere die Tapferkeit: Selbst wenn der Zorn uns Mut verleiht, so sind doch nicht wir es, die führen, wir werden geführt. Auch in dieser Kritik finden sich Reste der Ethik der inneren Regierung. Montaigne erweist sich hier in gewisser Weise als Schüler der Stoa, deren Emotionstheorie auf zwei Thesen pocht: Emotionen gehören zur rationalen Seele und die Seele ist eine Einheit. ${ }^{28}$ Für die meisten Stoiker sind Emotionen Urteile, mithin Handlungsgründe. Allerdings sind Emotionen schlechte Handlungsgründe, denn sie übernehmen gegen überlegte und bessere Handlungsgründe die Regierung. Wer sich von Emotionen leiten lässt, verrät Charakterschwäche. Diese These weist Montaigne zurück. Warum ist die zweite These, die Einheit der Seele, wichtig? Ihr zufolge gehören Emotionen keinem irrationalen Seelenteil an, der von der Vernunft verschieden wäre, sondern der ganzen Seele. Emotionen gehören zu uns, d. h. wir sind für sie verantwortlich. Die Stoiker wollen verhindern, dass wir Emotionen einem anderen Seelenteil zuschreiben, für den wir „nichts können“. Emotionen überkommen uns, reißen uns mit, machen uns zu schaffen, sie gehören gewissermaßen nicht zu uns. Dennoch wird die Verantwortung für Emotionen nicht aufgegeben, denn für Montaigne steht dieser Umstand der Verantwortung nicht entgegen, können wir doch auch Verantwortung für andere - Tiere, Kinder, Freunde oder Schüler - übernehmen. Diese Übernahme ist ein Rest der Ethik der inneren Regierung.

\section{La Rochefoucauld: Emotionen als Ausprägungen der Eigenliebe}

Während Montaigne Emotionen in einen moralphilosophischen Rahmen einordnet, betrachtet La Rochefoucauld sämtliche Emotionen als Ausprägungen der Eigenliebe (amour propre). Dadurch hat La Rochfoucaulds Nachdenken über Emotionen eine stärker systematische Basis als das an Beispielen orientierte Vorgehen Montaignes. Auch La Rochefoucauld setzt sich kritisch mit der Ethik der inneren Regierung auseinander, jedoch

28 Annas 1992, 103-120. 
auf ganz andere Art und Weise, wie die folgende, wichtige Maxime zeigt: „Der Mensch fühlt sich oft führend, wo er geführt wird; und während er durch seinen Geist [esprit] zu einem Ziel strebt, zieht ihn sein Herz [coeur] unmerklich zu einem anderen.“ (M 43)

Die innere Regierung ist eine der vielen Formen der Selbsttäuschung, um deren Entlarvung es den Moralisten geht. ${ }^{29}$ Sie ist lediglich eine Ausprägung der Eigenliebe. Was aber ist Eigenliebe? Sie sollte nicht mit Egoismus verwechselt werden. „Die Eigenliebe ist die Liebe zu sich selbst und zu allen Dingen für sich selbst.“ (M 563) In der Eigenliebe liebt sich das Subjekt und die anderen Dinge nur in Beziehung auf sich selbst. Diese Selbstbezüge sind jedoch nicht transparent, denn das Subjekt wird von der Eigenliebe geführt, wenn es sich zu führen glaubt. Die Eigenliebe ist ein anderes Subjekt (coeur) im Menschen, das Ziele verfolgt, die dem Geist (esprit) verborgen bleiben (M 102). Sie ist eine Schmeichlerin (M 2) und klüger als der klügste Mann (M 4). Sie ist eine zweite Person, die wir nicht kennen (M 3): „Man ist bisweilen von sich selbst so verschieden wie von anderen." (M 135). ${ }^{30}$ Die Eigenliebe treibt uns an und bleibt uns verborgen. ${ }^{31}$

Dies wurde mit Spinozas Conatus-Theorie verglichen. ${ }^{32}$ Der Vergleich ist hilfreich. Spinoza zufolge ist das Streben eines Dings, sich im Sein zu erhalten, dessen Wesen. ${ }^{33} \mathrm{Im}$ Falle des Menschen ist dieses Streben die Begierde (appetitus) und Emotionen sind Hindernisse oder Steigerungen dieses Strebens. ${ }^{34}$ Wir können uns die Eigenliebe als ein Streben vorstellen, dass die Essenz des Menschen ausmacht. Die Interpretation, die den folgenden Ausführungen zugrunde liegt, lautet, dass Eigenliebe das Streben ist, die Selbstachtung zu erhalten und zu steigern. Die Erhaltung der Selbstachtung aber fordert, dass dieses Streben sich teilweise vor sich selbst verbirgt, denn Beeinträchtigungen der Selbstachtung müssen unerkannt repariert werden, andernfalls würde dem Subjekt die Schädigung der Selbstachtung bewusst, was deren Erhaltung und Steigerung abträglich

29 Vgl. Starobinski 1966.

30 Vgl. die Deutung des Freudschen Unbewussten als zweite Person bei Rorty 1991.

31 Zu dieser „Doppelfunktion“ Stackelberg 1982, $131 \mathrm{f}$.

32 Charles-Marc Des Granges: „Cet amour-propre, c'est ce que Spinoza appelera: la tendance de l'être a persévérer dans l'étre. C'est une condition de notre existence physique et morale [...] elle est à l'homme ce qu'est la pesanteur ou l'attraction dans le monde physique: elle crée l'équilibre social.“ (Zitiert nach Stackelberg 1982, 133.)

33 Vgl. Spinoza, Ethik III p 7.

34 Vgl. den Beitrag zu Spinoza in diesem Band. 
wäre. Eigenliebe ist also das prä-reflexive Streben nach Erhaltung und Steigerung der Selbstachtung.

Die Emotionen nun sind spezifische Ausprägungen der Eigenliebe: „Die Leidenschaften sind nichts als die verschiedenen Vorlieben [les divers goûts] der Eigenliebe.“ (M 531) Weil die Eigenliebe die Liebe zu sich ist, ist das Selbst das allgemeinste (intentionale) Objekt von Emotionen. Alle Emotionen sind somit Mischformen, denn stets gehört die Liebe zu sich selbst dazu. La Rochefoucauld zufolge generiert das Herz, Sitz der Eigenliebe, unablässig Emotionen. Auch La Rochefoucauld betont die Autonomie der Emotionen (M 5), wichtiger ist aber deren Wandelbarkeit. Die Zerstörung einer Emotion bedeutet die Entstehung einer neuen (M 10), und oftmals bringen Emotionen deren Gegenteil hervor (M 11). Die Wandelbarkeit von Emotionen nun dient der Erhaltung der Eigenliebe, d. h. die Funktion von Emotionen besteht in der Erhaltung und Steigerung der Selbstachtung.

Der Aufbau der folgenden Maxime ist typisch für La Rochefoucauld: „Wir können nichts lieben, außer in Beziehung auf uns, und wir folgen nur unserer Vorliebe und unserem Vergnügen, wenn wir unseren Freunden uns vorziehen; nichtsdestotrotz kann die Freundschaft allein aufgrund dieser Bevorzugung wahr und vollkommen sein.“ (M 81)

Die Liebe zu einer Person wird als Selbstbezogenheit charakterisiert. Liebe ist, wie alle Emotionen, primär ein Selbstverhältnis und Ausdruck der Eigenliebe. Der Bezug der Eigenliebe auf andere wird dadurch hergestellt, dass wir am anderen dasjenige lieben, was unseren „Vorlieben“ entspricht und uns „Vergnügen“ bereitet (M 88). Warum sollten wir ihn sonst auch lieben? Dies tun wir selbst dann und gerade dann, wenn wir den geliebten Freund uns gegenüber bevorzugen, beruht dies doch auf unseren Vorlieben. Der springende Punkt ist, dass in der Liebe zu einem Freund jene Qualitäten affirmiert werden, die wir an uns selbst achten. So ist Freundschaft eine Steigerung unserer Selbstachtung. ${ }^{35}$ Dies alles beruht

35 Hier liegt der Einwand nahe, dass wir an anderen doch auch Qualitäten achten können, die uns fehlen. Eine Antwort im Sinne La Rochefoucaulds lautet, dass wir die fragliche Qualität wünschen, sie aber nicht haben oder erwerben können, und sie deshalb am anderen schätzen. Der andere kompensiert unseren Mangel. Wie steht es jedoch mit Qualitäten, die wir einfach um ihrer selbst willen an einem anderen achten, ohne sie überhaupt zu wünschen? Hier handelt es sich in La Rochefoucaulds Sicht natürlich um Formen der Selbsttäuschung: Das Problem einer Qualität, die wir an anderen schätzen, die wir für uns jedoch wünschen, aber nicht haben, besteht für die Eigenliebe darin, dass sie unsere Selbstachtung herabsetzt. Eine Möglichkeit, dies vor uns zu verbergen, ist die Idealisierung der Quali- 
auf Gegenseitigkeit (M 83). Vielen Emotionen kommt eine soziale Rolle zu, d. h. wir sind in ihnen auf andere bezogen. Dieser Bezug ist stets Ausdruck der Eigenliebe. Nur durch die Bevorzugung jener Qualitäten am Freund, die wir an uns selbst schätzen, kann die Freundschaft „wahr und vollkommen" sein. Allerdings muss die Eigenliebe verborgen bleiben, damit die Beziehung auf den anderen für das Subjekt erhalten bleibt. Betrachten wir zur Verdeutlichung dieses Aspekts eine weitere Emotion.

Eine der heftigsten Formen der Traurigkeit ist die Trauer über den Verlust einer geliebten Person. Wir weinen, weil wir traurig über den Verlust sind, weil wir um den anderen trauern. In der Trauer beziehen wir uns auf ein Objekt (die Person) unter einem bestimmten Aspekt (Verlust) und die Spannung zwischen Objekt und Aspekt äußert sich in Tränen. La Rochefoucauld konterkariert dieses Bild der Traurigkeit mit dem Verdacht auf Heuchelei. Er unterscheidet vier Formen der Heuchelei (M 233):

(1) Wir beweinen nicht den anderen, sondern bedauern das Verschwinden der guten Meinung, "die er von uns gehabt hat" und die daraus folgende „Verringerung unseres Wohlergehens, unseres Vergnügens". Dabei täuschen wir uns selbst über diese Motive. Aufgrund der Selbsttäuschung handelt es sich um Heuchelei.

(2) Wir trauern nicht um den anderen, sondern nehmen den Verlust zum Anlass, uns eine neue Persona zu bilden. Laut La Rochefoucauld befällt diese Form der Heuchelei ehrgeizige Frauen, denen es verwehrt ist, Ruhm auf anderen Wegen zu finden. Sie stilisieren ihr Leben zum Schauspiel eines unüberwindlichen Verlusts und versuchen „in allen ihren Handlungen davon zu überzeugen, dass ihr Leid erst mit ihrem Leben enden wird“".

(3) Wir weinen, um als zart zu gelten, um bemitleidet und beweint zu werden.

(4) Wir weinen, um dem Vorwurf zu entgehen, nicht geweint zu haben.

Dass wir uns traurig fühlen, steht außer Frage. Aber wir geben vor und glauben auch, die Motivation unserer Traurigkeit sei der Verlust einer Person. Die Trauer ist dem aktuellen Selbst- und Fremdbezug nachgeordnet, wobei für La Rochefoucauld die Eigenliebe ausschlaggebend ist: „Wel-

tät des anderen, von der wir uns sagen, dass wir sie einfach um ihrer selbst willen achten. 
chen Vorwand wir unserer Betroffenheit auch immer geben mögen, oft werden sie durch nichts als Eigennutz und Eitelkeit [l'intérêt et la vanite] verursacht.“ (M 232) Die Heuchelei hat Vorteile: Die Emotion behält die Ausrichtung auf den Verstorbenen oder auf das soziale Umfeld. In der Ausrichtung auf das soziale Umfeld dient die Trauer der kurzfristigen Erhaltung der Selbstachtung (4), der kurzfristigen Steigerung der Selbstachtung (3) oder der langfristigen Erhaltung und Steigerung der Selbstachtung (2). Was ist mit Fall (1)? Nun, wir bedauern den Verlust einer Quelle der Selbstachtung, da wir im Verstorbenen unsere an uns bevorzugten Qualitäten liebten. Es ist dieser Verlust, den das Subjekt um der Selbstachtung willen vor sich selbst verbergen muss.

Es gibt Emotionen, die unserer Selbstachtung unmittelbar abträglich sind, wie der Neid. Man könnte einwenden, dass der Neid ein Gegenbeispiel zu der These darstelle, Emotionen hätten die Erhaltung und Steigerung der Eigenliebe zum Ziel, denn er fördere die Selbstachtung gerade nicht, führe er dem Neider doch einen seiner Mängel vor Augen, nämlich das Objekt des Neids. Aber auch für den Neid muss gelten, dass er der Eigenliebe entspringt, gerade weil seine Ursache in der Verletzung der Selbstachtung liegt. Und diese muss durch die Verwandlung des Neids mittels anderer und in andere Emotionen wiederhergestellt werden. Wie geht das? Neid ist „eine Raserei, die die Güter der anderen nicht erträgt.“ (M 28). Er ist unversöhnlicher als Hass (M 328) und erhält sich über das Objekt des Neides („,das Glück dessen, den wir beneiden“) hinweg seine Autonomie (M 476). Tritt er offen zutage, droht uns gar die Verachtung anderer: „Man hausiert oft sogar mit den verbrecherischsten Leidenschaften, doch der Neid ist eine schüchterne und beschämende [timide et honteuse] Leidenschaft, die zuzugeben man sich nicht traut.“ (M 27)

Wie kann der Neid verborgen und die Selbstachtung erhalten werden? Nun, er wird umgewandelt und dadurch die Selbstachtung erhalten. Neid auf die Güter arrivierter Personen wird zur freudigen Akklamation von Neulingen (M 280). Mäßigung ist eine Furcht vor dem Neid, den diejenigen auf sich ziehen, die sich an ihrem Glück berauschen (M 18). Stolz, der uns zu vielen neidischen Regungen veranlasst, versteht es zugleich, ihn zu mäßigen (M 281). Betrachten wir ein abstraktes Beispiel: ${ }^{36} \mathrm{~A}$ hat X und $\mathrm{B}$ möchte X. B verspürt Neid. Dieser Neid verletzt Bs Selbstachtung, denn $\mathrm{B}$ hat weniger als $\mathrm{A}$ und zudem ist der Neid eine „beschämende Leiden-

36 In Anlehnung an Elster 1999, 98f., der sich an La Rochefoucauld und la Bruyère orientiert. 
schaft". Neid wird zu Demut und Scham. Um demgegenüber die Selbstachtung zu erhalten, wird der Neid umgedeutet. Dies geschieht z. B. dadurch, dass B die Relation zwischen X und A oder seine Emotion umdeutet. A hat X nicht verdient und B ist Stolz auf seine Entsagung; A hat sich $\mathrm{X}$ auf zweifelhaften Wegen verschafft, und B ist empört; A versteht es nicht, X zu nutzen, und B fühlt Überlegenheit. Stolz, Empörung oder Überlegenheit sind jeweils das Resultat von Umwandlungen zum Zweck der Erhaltung der Selbstachtung. Am Beispiel des Neids wird nicht nur deutlich, dass Emotionen der Eigenliebe entspringen, die Funktion haben, diese zu erhalten und zu steigern, und dass die entsprechenden Umwandlungen dem Subjekt verborgen bleiben müssen, sondern auch, dass sie mit anderen Emotionen, mit Überzeugungen und Wünschen interagieren.

\section{Autonomie der Emotionen und Emotionen als Handlungen}

Jon Elster hat betont, dass bei den Moralisten die Emotionen als Objekte einer Theorie psychologischer Mechanismen in den Blick geraten. Er ist der Ansicht, die Moralisten hätten in der Geschichte der Emotionstheorien einen wichtigen Schritt genommen, indem sie sich auf die Rolle der Emotionen im sozialen Raum und im Subjekt konzentriert hätten. Mit seinem Ansatz habe etwa La Rochefoucauld einen theoretischen Rahmen für die Untersuchung der Interaktion zwischen verschiedenen Emotionen, Wünschen und Überzeugungen, d. h. deren gegenseitige Hervorbringung und Verwandlung, geschaffen. ${ }^{37}$ Was sind die Gründe dafür, dass dies bei den Moralisten geschieht? Pascal schreibt über die Form seiner Gedanken: „Ich erweise meinem Gegenstand zu viel der Ehre, wenn ich ihn auf ordentliche Weise behandle, denn ich will zeigen, dass er der Ordnung unfähig ist." 38 Wie bei Montaigne ist der unordentliche Gegenstand der Mensch. Beim Religionsphilosophen Pascal geht es jedoch nicht um den Menschen tout court, sondern um den Menschen ohne Beistand der christlichen Religion, dessen Darstellung eines der Hauptziele der anthropologischen Reflexionen der Pensées ist. Es sind vor allem die Emotionen, die in Unordnung sind und den Menschen in Unordnung halten, sie sind „tousjours [sic!] déreglées“, wie etwa Jean-François Senault (1599-1672) meint.

37 Vgl. Elster 1999, 76-107.

38 Pascal 1991 (posthum zuerst 1670), 365 (Nummerierung Selliers 457). Übersetzung des Verfassers. 
Für Senault ist die Unordnung der Emotionen u. a. eine Folge des Sündenfalls. Ohne Gottes Gnade kann sie die Vernunft nicht befrieden und regulieren. ${ }^{39}$ Die neostoische Idee einer Kontrolle der Emotionen ohne göttliche Gnade, die verblendete Idee einer laizistischen Weisheit, ist nur ein anderer Ausdruck dieses deplorablen Zustands. Dieser religionsphilosophische Hintergrund spielt auch bei Montaigne und La Rochefoucauld eine gewisse Rolle. So verweist Montaigne darauf, dass er den Menschen beschreibt, wie er ohne göttlichen Beistand ist: nackt, unstet, und überheblich (E II, 12). La Rochefoucaulds Maximen enthalten zwar keinerlei Hinweise auf religiöse Kontexte, doch die zeitgenössischen Leser stellten solche Verbindungen umstandslos her. ${ }^{40}$ Wie auch immer man die Relation zwischen der Religion und den Emotionen bei Montaigne oder La Rochefoucauld verstehen möchte, ${ }^{41}$ wichtig ist, dass sie die Emotionen aus dem religiösen Kontext lösen und dadurch zu eigenständigen Objekten der Betrachtung machen. Wie eingangs gesagt, betrachten Moralisten Emotionen in Selbst- und Fremdverhältnissen, Autoren wie Montaigne und La Rochefoucauld gestehen einzelnen Emotionen nicht nur Autonomie relativ zu Überzeugungen oder Wünschen zu, sondern auf einer weiteren Ebene auch Autonomie relativ zu naturwissenschaftlichen oder metaphysischen und vor allem zu theologischen Projekten.

Ein Grundzug gegenwärtiger Emotionstheorien besteht darin, Emotionen in Analogie zu anderen mentalen Vermögen zu betrachten. Man kann diese Theorien danach gruppieren, ob sie Emotionen eher wie Körperwahrnehmungen (Empfindungstheorien), wie Urteile (kognitive Theorien), wie Sinneswahrnehmungen (Wahrnehmungstheorien) oder wie Organe mit Funktionen (evolutionäre Theorien) behandeln. ${ }^{42}$ Lebewesen verfügen jedoch nicht nur über sensitive, perzeptive oder kognitive Vermögen, sondern auch über Handlungsfähigkeit. Der implizite Vorschlag der Moralisten lautet, Emotionen eher wie Handlungen zu betrachten.

39 Senault 1987, 88-89; vgl. Levi 1964, 19.

40 Vgl. Parmentier 2000, 67f.

41 Die Eigenliebe wird bisweilen mit der augustinischen amor sui in Verbindung gebracht, mit der sündigen Selbstbezogenheit des Menschen, der Gott und darüber sein Heil vergisst. Doch die Eigenliebe entbehrt in den Maximes jedweder theologischen Einbettung. Zum theologischen Kontext vgl. Levi 1964, 203-233. Gegen die in Lafont 1977 vertretene These eines augustinischen La Rochefoucauld vgl. m. E. überzeugend Stackelberg 1982, 104-108; Clark 1994, 105-120. Anders Parmentier 2000, 65-72.

42 Vgl. de Sousa 2003. 
Wie ist dies zu verstehen? Wie wir gesehen haben, verweist Montaigne auf die Möglichkeit eines gezielten und dramaturgischen Umgangs mit Emotionen. Er hebt bereits im Beispiel der zornigen Eltern eine gewisse „Theatralität" des Zorns hervor, wenn es heißt, er wünschte, „mit bühnenreifem Donnerwetter dreinzufahren und den Ärmsten zu rächen“. Viele Emotionen haben eine dramatische Struktur. Wir führen sie gleichsam als Theaterstücke auf, etwa wenn wir mit einer Situation nicht zurechtkommen, wenn diese uns überfordert oder unsere Selbstachtung direkt oder über den Weg der Verachtung durch andere verletzt. La Rochefoucaulds Analysen der geheuchelten Trauer gehen in dieselbe Richtung, ebenso die Manöver zur Erhaltung der Selbstachtung im Neid. Emotionen sind also so etwas wie gespielte Handlungen. Diese Handlungen können kurz und komprimiert (ein Schlag auf den Tisch, ein tiefer Seufzer) oder lang und komplex sein, wie etwa Elternliebe oder Hass. Die Metapher einer dramatischen Handlung vermag unterschiedlichste Emotionen zu vereinen. Emotionen zeichnen sich jedoch auch durch körperlich expressive und phänomenale Manifestationen aus. Als Manifestationen unterscheiden sich Emotionen vom bloßen Theaterspiel, denn durch die Manifestationen sind wir unmittelbar affiziert. Deshalb glauben wir tatsächlich an die dramatische Handlung. Die mimetische Übertragung der expressiven Manifestationen bezieht andere in die dramatische Handlung mit ein. Darin gleichen Montaignes und La Rochefoucaulds Ansätze JeanPaul Sartres Emotionstheorie. ${ }^{43} \mathrm{Ihr}$ zufolge sind Emotionen „eine Transformation der Welt" “44 Emotionen sind Reaktionen, durch die wir ein psychologisches Gleichgewicht herstellen, indem wir unsere Wahrnehmung einer Situation und nicht diese selbst ändern (sie transformieren). Sartre verweist zur Veranschaulichung auf La Fontaines Fabel der sauren Trauben: Wir wünschen uns die Trauben, können sie aber nicht erreichen, also ändern wir die Wahrnehmung der Trauben und murmeln: „Sie sind noch zu grün". ${ }^{45}$ Ganz ähnlich schreiben wir im Zorn einer Person die Eigenschaft zu, verletzend oder hassenswert zu sein, weil sie der Befriedigung unserer Wünsche im Wege steht.

Die Analogie wirft Probleme auf. Zuerst kann man darauf hinweisen, dass Handlungen eine teleologische Struktur haben weil sie Absichten ent-

43 Zur Analogie mit Handlungen vgl. Sartre 1997 (franz. zuerst 1939), 304f. Vgl. den Beitrag zu Sartre in diesem Band.

44 Sartre 1997, 294.

45 Sartre 1997, 295f. 
springen und Ziele verfolgen. La Rochefoucaulds These jedoch gibt den Emotionen die Erhaltung und Steigerung der Selbstachtung zum Ziel. Wie steht es jedoch mit den Absichten? Handlungen werden ja von jemandem ausgeführt, sie haben einen Urheber, dem sie zugerechnet werden. Etwas ist u. a. erst dann eine Handlung, wenn es die direkte Folge eines intentionalen Zustands eines Subjekts ist. Demgegenüber wären Emotionen jedoch Handlungen, die wir nicht aus-, sondern aufführen, so dass wir uns gar nicht als Urheber dieser Handlungen verstehen. Emotionen werden erlitten, man kann nicht beliebig aus ihnen heraus, es sind Passionen. La Rochefoucaulds Antwort lautet, wie wir gesehen haben, dass wir mit der Eigenliebe ein anderes Subjekt (coeur) postulieren müssen, das Ziele verfolgt, die dem Geist (esprit) verborgen bleiben. Sie ist eine Art zweiter Person, die wir nicht kennen. La Rochefoucaulds Antwort sollte man als Folge von Montaignes moralpsychologischer Idee betrachten, Emotionen als etwas uns Fremdes zu betrachten, das uns gleichsam überkommt, für das wir jedoch die Verantwortung übernehmen können, sodass uns Emotionen zugerechnet werden dürfen. Was auch immer man von diesem Vorschlag halten mag, er ist beachtlich, weil er eine Umkehrung des gewöhnlichen Verständnisses der Emotionen als Passionen bedeutet, und weil er einige Phänomene in unserem emotionalen Leben zu beschreiben vermag.

\section{Literatur}

Die Essais werden nach Montaigne 1965 zitiert, mit Angabe von Buch, Essay, frz. Seite/dt. Seite (die Übersetzungen stammen von Hans Stilett, siehe Montaigne 1998). Die Maximes von La Rochefoucauld werden nach den Oeuvres complètes zitiert unter Angabe von M und der entsprechenden Nummerierung der Maxime. Die Übersetzungen stammen vom Verfasser. - Vollständige Angaben siehe unten. Die verwendeten Siglen sind:

E - Montaigne, Essais

M - La Rochefoucauld, Maximes

Annas, Julia E. (1992), Hellenistic Philosophy of Mind, Berkeley.

Balmer, Hans Peter (1981), Philosophie der menschlichen Dinge. Die europäische Moralistik, Bern.

Campion, Pierre (1998), Lectures de La Rochefoucauld, Rennes.

Clark, Henry C. (1994), La Rochefoucauld and the Language of Unmasking in Seventeenthcentury, Genf.

Elster, Jon (1999), Alchemies of the Mind. Rationality and the Emotions, Cambridge.

Evans, Dylan (2002), The Search Hypothesis of Emotion, in: British Journal for the Philosophy of Science 53, 497-509. 
Goldie, Peter (2000), The Emotions. A Philosophical Exploration, Oxford.

Griffiths, Paul E. (1997), What Emotions Really Are. The Problem of Psychological Categories, Chicago.

Hallie, Philip (1977), The Ethics of Montaigne's De la curauté, in: Raymond La Charité (Hg.), Ob un amy!, Lexington, 156-171.

Kruse, Margot (2003), Beiträge zur fran₹ösischen Moralistik, hrsg. von Joachim Küpper, Berlin.

Lafond, Jean (1977), La Rochefoucauld. Augustinisme et littérature, Paris.

Lafond, Jean (Hg.) (1992), Moralistes du XVIIe siècle, Paris.

La Rochefoucauld, François duc de (1964, zuerst 1664ff.), Oeuvres complètes (edition établi par Louis Martin-Chauffier, introduction par Robert Kanters), Paris (= M).

Levi, Anthony (1964), French Moralists. The Theory of the Passions 1585 to 1649, Oxford.

Lipsius, Justus (1965, lat. zuerst 1583/84), Von der Bestendigkeit/De Constantia (Faksimiledruck der dt. Übersetzung des Andreas Viritius 1601, hrsg. von Leonard Forster), Stuttgart.

Montaigne Michel de (1965, zuerst 1580), Les Essais, hrsg. von Pierre Villey und Verdun-Louis Saulnier, Paris (= E).

Montaigne Michel de (1998, franz. zuerst 1580), Essais (übers. von Hans Stilett), Frankfurt a. M. (= E).

Parmentier, Bérengère (2000), Le siècle des moralistes. De Montaigne à La Bruyère, Paris.

Pascal, Blaise (1991, posthum zuerst 1670), Pensées, hrsg. von Philippe Sellier, Paris.

Quint, David (1998), Montaigne and the Quality of Mercy, Princeton.

Ricoeur, Paul (1993, franz. zuerst 1965), Die Interpretation. Ein Versuch über Freud, Frankfurt a. M.

Rorty, Richard (1991), Freud and Moral Reflection, in: ders., Essays on Heidegger and Others, Cambridge, 143-163.

Sartre, Jean-Paul (1997, franz. zuerst 1939), Skizze einer Theorie der Emotionen, in: ders., Die Transzendenz des Ego. Philosophische Essays 1931-1939, Reinbek bei Hamburg, 255-321.

Schneewind, Jerome B. (1998), The invention of autonomy. A history of modern moral philosophy, Cambridge.

Senault, Jean-François (1987, zuerst 1641), De l'usage des passions, Paris.

Sousa, Ronald de (2003), Emotion, in: Edward N. Zalta (Hg.), The Stanford Encyclopedia of Philosophy (Spring 2003 Edition):

URL: http://plato.stanford.edu/archives/spr2003/entries/emotion/ (letzter Zugriff: 13.07.2008).

Stackelberg, Jürgen von (1982), Franæösische Moralistik im europäischen Kontext, Darmstadt.

Starobinski, Jean (1966), La Rochefoucauld et les morales substitutives, in: La nouvelle revue françaises $28,16-34$.

Wild, Markus (2006), Die anthropologische Differen\%: Der Geist der Tiere in der frühen Nenzeit bei Montaigne, Descartes und Hume, Berlin.

Zimmer, Robert (1999), Die europäischen Moralisten zur Einführung, Hamburg. 